\title{
Acquisition de la prosodie en langue additionnelle : l'accentuation en français par des locuteurs adultes plurilingues de langue initiale turque
}

\author{
Aline Marchand ${ }^{1, *}$, et David Le Gac ${ }^{2}$ \\ ${ }^{1}$ DyLiS, Université de Rouen Normandie, 76830 Mont Saint Aignan Cedex, France \\ ${ }^{2}$ DyLiS, Université de Rouen Normandie, 76830 Mont Saint Aignan Cedex, France
}

\begin{abstract}
Résumé. Cette étude expérimentale porte sur l'acquisition des accents primaire $(A 1)$ et secondaire $(A 2)$ en français langue additionnelle $(L a)$ par des locuteurs adultes plurilingues de langue initiale $(L i)$ turque. Étant donné les convergences et divergences entre les A1 et A2 français et turcs concernant leurs places et paramètres acoustiques, l'objectif est de voir comment sont réalisés ces accents en français La par les locuteurs turcs. L'analyse contrastive des deux systèmes accentuels suggère que, par rapport au français $\mathrm{Li}$, les locuteurs turcs réalisent des écarts phonétiques plutôt que phonologiques. L'analyse acoustique avec Praat confirme nos hypothèses : sur le plan phonologique, l'A1 en français La est bien placé en fin de groupe accentuel, avec les paramètres acoustiques pertinents $\left(\mathrm{F}_{0}\right.$ et durée), et l'A2 initial n'est pas réinterprété comme un A1 turc non final; mais sur le plan phonétique, les locuteurs ont des difficultés à gérer les proportions des allongements et des variations de $F_{0}$. Ces résultats confirment les prédictions de l'Hypothèse de la surdité accentuelle (Dupoux \& al. 1997/2010) et de l'Hypothèse de la Différence de Marque (Eckman 1977/2008) : l'A1 turc étant au moins aussi complexe que l'A1 français, les locuteurs turcs ne sont pas sourds aux différences de l'A1 français.
\end{abstract}

\begin{abstract}
Acquisition of prosody in an additional language: the accentuation in French by multilingual adult speakers of Turkish as initial language. This experimental study is about the acquisition of the primary (A1) and secondary (A2) accents in French as an additional language $(a L)$ by multilingual adult speakers of Turkish as initial language $(i L)$. Given the similarities and differences between the French and Turkish A1 and A2 regarding their places and acoustic features, the aim is to examine how these accents are realized in French aL by the Turkish speakers. The contrastive analysis of the accentual system of both languages suggests that, compared with French iL, the Turkish speakers realize more phonetic differences than phonological ones. The acoustic analysis with Praat confirms our hypotheses: at the phonological level, the French A1 is correctly located at the end of the accentual phrase with the
\end{abstract}

\footnotetext{
*Corresponding author : aline.marchand2@univ-rouen.fr
} 
relevant acoustic features ( $\mathrm{F}_{0}$ and duration), and the initial $\mathrm{A} 2$ is not reinterpreted as a Turkish non final A1; but at the phonetic level, the speakers have some difficulties to achieve the correct lengthening and $\mathrm{F}_{0}$ variations. These results confirm the predictions of the stress deafness Hypothesis (Dupoux \& al. 1997/2010) and of the Markedness Differential Hypothesis (Eckman 1977/2008): as the Turkish A1 is at least as complex as the French A1, the Turkish speakers can hear the differences of the French A1.

\section{Contexte, enjeux et objectifs}

\subsection{Contexte et enjeux : l'acquisition de la prosodie en langue additionnelle}

L'importance de la prosodie dans la parole est aujourd'hui largement reconnue (Di Cristo 2016). En effet, l'utilisation adéquate des traits segmentaux, i.e. la perception des phonèmes et la production des sons, ne suffit pas à communiquer de façon efficace. Les éléments prosodiques, i.e. l'accentuation, l'intonation et les phénomènes temporels tels que le rythme et les pauses, sont prépondérants, à la fois en production et en perception, car ils véhiculent des signifiés lexicaux et supra-lexicaux (Ladd 1996/2008, Post 2000): on pense par exemple à l'opposition bien connue du contour mélodique montant vs descendant, qui permet d'indiquer la modalité intonative des énoncés (interrogative vs déclarative). De ce fait, la recherche a montré un intérêt croissant depuis 20 ans pour l'acquisition de la prosodie en langue additionnelle ( $L a$ ). Cependant, ces études ont concerné majoritairement l'acquisition de l'anglais, par des locuteurs de différentes langues initiales $(\mathrm{Li})$ : espagnol (Zubizarreta \& Nava 2011), français (Horgues 2010), vietnamien (Nguyen \& Ingram 2005)... Certes, les La étudiées se diversifient : polonais (Kijak 2009), espagnol (Dupoux \& al. 1997, 2001, 2008, 2010), français (Duběda 2012, Schwab 2012, Santiago 2016), français et néerlandais (Rasier \& Hiligsmann 2007)... Mais une seule étude à notre connaissance porte sur le français parlé par des locuteurs adultes de Li turque de Turquie (Y1lmaz 2004), et c'est une étude très succincte et non expérimentale. Par ailleurs, il y a eu depuis cinq à six décennies un essor des modèles théoriques dans le domaine de l'acquisition de la prononciation d'une La: l'Hypothèse de l'Analyse Contrastive (Lado 1957, ...), l'Hypothèse de l'Interlangue (Selinker 1972), l'Hypothèse de la Différence de Marque (Eckman (1977/2008), l'hypothèse de la surdité accentuelle (Dupoux \& al. 1997/2010), parmi d'autres. Toutefois, la plupart de ces modèles ont été appliqués au domaine segmental surtout, mais très peu à la prosodie. Notre étude pilote consiste donc à analyser la réalisation de deux phénomènes prosodiques importants en français La par des locuteurs adultes de Li turque, à savoir l'accent primaire (A1) et l'accent secondaire (A2).

La notion d'accent est très polysémique et dépend pour beaucoup du cadre théorique et du niveau d'analyse où l'on se situe (cf. Van der Hulst 2012, Di Cristo 2016). Dans le présent article, l'accent renvoie à l'utilisation des paramètres prosodiques, i.e. surtout la fréquence fondamentale $\left(F_{0}\right)$ et la durée, pour mettre en relief certaines syllabes. L'accentuation est une des composantes fondamentales de la prosodie. Par exemple, le mauvais placement de l'accent entraîne des problèmes de compréhension (Hahn 2004) et des ruptures de communication (Jenkins 2002, Derwing \& Munro 2009), tandis qu'un placement correct de l'accent entraîne au contraire une bonne compréhension des énoncés de La (Field 2005).

Les travaux sur l'acquisition de la prosodie en La étudient souvent le transfert dans la La des unités phonologiques de la Li et de leurs réalisations phonétiques, qu'il s'agisse d'un transfert positif ou négatif. Ces études partent d'une analyse contrastive entre $\mathrm{Li}$ et $\mathrm{La}$. La version prédictive de l'Hypothèse de l'Analyse Contrastive (HAC : Lado 1957) estime que 
les phénomènes linguistiques semblables ou proches en $\mathrm{Li}$ et en $\mathrm{La}$ sont acquis plus facilement (transfert positif), par opposition aux unités linguistiques nouvelles ou différentes, censées être plus difficiles à acquérir (d'où un risque de transfert négatif). La version explicative de l'HAC, elle, explique leurs difficultés par l'Analyse des Erreurs ( $A E$ : Corder 1967/1974). Enfin, la version modérée de l'HAC (Wardhaugh 1970, Brown 1987, Flege 1992) montre que les entités nouvelles ou différentes sont parfois acquises plus facilement que les traits linguistiques ressemblants. L'Hypothèse de l'Interlangue (Selinker 1972), elle, montre que l'AE ne prend pas en compte les formes correctes en La : or, il n'y a pas seulement des questions de transfert positif et négatif, mais aussi une sur- ou sousutilisation en La des traits prosodiques de la Li. Quant à Eckman (1977/2008), il reprend la théorie de la Marque, selon laquelle une unité linguistique est plus marquée qu'une autre si elle a au moins un trait de plus, et propose l'Hypothèse de la Différence de Marque (HDM : 1977/2008) : le degré de difficulté est proportionnel au degré de la Marque. Donc les phénomènes marqués de La qui sont plus marqués que les phénomènes marqués de Li sont plus difficiles à apprendre, tandis que les phénomènes marqués de La qui sont moins marqués que les phénomènes marqués de Li sont plus faciles à apprendre. Les phénomènes marqués de Li ont moins de risques d'être transférés que les non marqués. Enfin, l'hypothèse de la surdité accentuelle (stress deafness : Dupoux \& al. 1997/2010) montre que les locuteurs sont sourds aux phénomènes prosodiques qui sont distinctifs dans la La, mais qui ne le sont pas dans leur Li.

Les études sur l'acquisition de la prosodie en La montrent que les locuteurs ont des difficultés similaires, quelles que soient leurs $\mathrm{Li}$ et $\mathrm{La}$, et que le transfert de la $\mathrm{Li}$ est un facteur important (cf. Mennen 2007 pour un passage en revue). D'une part, il peut y avoir des erreurs au niveau phonologique, i.e. les locuteurs font des erreurs concernant l'inventaire, la distribution, les formes et significations des unités phonologiques (problèmes de perception et/ou de production de la signification). Par exemple, ils ont des difficultés à placer l'accent au bon endroit ou l'utilisent trop souvent ou pas assez; ils produisent une hausse de $\mathrm{F}_{0}$ incorrecte sur des syllabes non accentuées, ... Et/ou, d'autre part, les locuteurs réalisent des variantes phonétiques à l'intérieur d'une même catégorie phonologique (cf. Rasier \& Hiligsmann 2007 pour un passage en revue) : une gamme de fréquences plus étroite que celle des locuteurs de $\mathrm{Li}$, un niveau global de fréquence plus haut ou plus bas, des pics mélodiques également plus hauts ou plus bas sur les syllabes accentuées, et/ou avec des différences d'alignement temporel, ...

En ce qui concerne l'acquisition de l'accent en français La, des recherches récentes montrent que plusieurs locuteurs sur-utilisent l'A1 (Schwab 2012) et l'A2 (Duběda 2012). Ainsi, sur le plan phonologique, les locuteurs espagnols accentuent trop de mots dans l'énoncé, suggérant un transfert négatif de l'accent lexical de l'espagnol (un par mot) en français ; sur le plan phonétique, ils réalisent un allongement trop important dans les syllabes accentuées et une $\mathrm{F}_{0}$ plus élevée que celle des locuteurs de français Li (Schwab 2012). Les locuteurs tchèques, au niveau phonologique, acquièrent facilement en français la distribution de 1'A1 final et de l'A2 initial, avec une perception plus aisée de l'A2 initial français et un transfert positif de l'accent initial tchèque ; ils ont même tendance à surutiliser 1'A2 français. Au niveau phonétique, ils réalisent un transfert négatif de certaines réalisations de l'accent initial tchèque, avec plus d'accents Bas (notés B*) et Bas*Haut (notés $\mathrm{B}^{*} \mathrm{H}$ ) ; mais les écarts phonétiques sont surtout quantitatifs, et dans les limites de la variabilité du français Li (Duběda 2012). La tendance à sur-utiliser l'accent est caractéristique du début d'apprentissage, quelles que soient la Li et la $\mathrm{La}$ (cf. Rasier \& Hiligsmann 2007 pour un passage en revue), et notamment pour les locuteurs néerlandais de français, mais on l'observe parfois aussi quand les locuteurs ont un assez bon niveau en La (Rasier \& Hiligsmann 2007). 


\subsection{Objectifs}

L'objectif principal de ce travail est d'étudier les réalisations accentuelles en français $\mathrm{La}$ par des locuteurs adultes plurilingues de Li turque, en nous concentrant sur l'accentuation dans le Groupe Nominal $(G N)$. Nous partirons d'une analyse contrastive des deux systèmes accentuels et nous nous demanderons en particulier dans quelle mesure les locuteurs turcs transfèrent en français les spécificités de l'A1 et de l'A2 du turc, et de quels types sont ces transferts. Dans un deuxième temps, nous présenterons les résultats d'une étude acoustique et expérimentale que nous avons menée pour répondre à ces interrogations. Enfin, ces résultats nous amèneront à discuter certaines des approches mentionnées précédemment sur l'acquisition de la prononciation d'une La.

\section{Analyse contrastive de l'accentuation du français et du turc}

Les similitudes et différences des A1 et A2 français et turcs concernent leur place et leurs paramètres acoustiques.

\subsection{L'A1 et l'A2 en français}

Sur le plan syntaxique, le français est une langue de structure SVO et le GN est constitué ainsi : un déterminant (Dét.) obligatoire sauf avec les Noms Propres (NP : *la Marie) et un Nom $(N)$. Ce N peut être modifié par un Groupe Adjectival (GAdj.) à sa gauche et par différents types de syntagmes à sa droite (GAdj., Relative/Participe, Groupe Prépositionnel : GPrép.). Ainsi dans le GN ci-dessous, le $\mathrm{N}$ tête maison est modifié par les GAdj. très grande, blanche, restaurée et par le GPrép. de nos voisins :

Ex. : La (très grande) maison (blanche) (restaurée) (de nos voisins)

$$
\text { [Dét. (GAdj.) N (GAdj.) (GAdj.) (GPrép.) }]_{\mathrm{GN}}
$$

$\mathrm{Au}$ niveau prosodique, un GN comprenant un $\mathrm{N}$ et un syntagme lexical à gauche ou à droite correspond généralement à un groupe prosodique particulier que nous appellerons Groupe Accentuel (GA) (Rossi 1999, Jun \& Fougeron 1995/2000). On a donc :

[Dét. (GAdj.) N (GAdj./GPrép.) $]_{\mathrm{GN}} \rightarrow$ [Dét. (GAdj.) N (GAdj./GPrép.) $]_{\mathrm{GA}}$

Le GA est généralement considéré comme le domaine de l'A1. L'A1 du français est un accent obligatoire, dont les indices acoustiques majeurs sont la fréquence et la durée (Rossi 1999, Di Cristo 1998/2016) ; donc il se caractérise par une durée plus longue et par une hausse ou une baisse mélodique (notée $\mathrm{H}^{*}$ pour un accent mélodique Haut ou B* pour un accent mélodique Bas), en fonction de l'intonation de l'énoncé. L'A1 est fixe, réalisé sur la dernière syllabe pleine (i.e. sans "e" muet) du dernier item lexical du GA (syllabe notée ici en majuscules et soulignée), assumant ainsi une fonction démarcative (Garde 1968, Fónagy 1979, Di Cristo 1998/2016, Rossi 1980/1999, Vaissière 1991).

Ex.: $\quad(\text { mon aMI })_{\mathrm{GA}} ;$ (mon ami d'enFANCe) $)_{\mathrm{GA}}$ (Rossi 1999).

Le GA peut aussi avoir un A2 facultatif, placé la plupart du temps sur la première syllabe ou parfois sur la deuxième du premier item lexical du GA (syllabe notée ici en majuscules seulement) et réalisé par une montée de $\mathrm{F}_{0}$, avec parfois un allongement de la consonne initiale de la syllabe, quand elle existe (Rossi 1999, Di Cristo 1998/2016). Il peut donc y avoir une alternance de syllabes accentuées et de syllabes inaccentuées au sein du GA (Verluyten 1984).

Ex.: $\quad(\text { orDInaTEUR })_{\mathrm{GA}}$

L'A2 a donc surtout une fonction démarcative, en reliant le début et la fin des unités lexicales et syntaxiques (arc accentuel (étendu) : Fónagy 1979). 


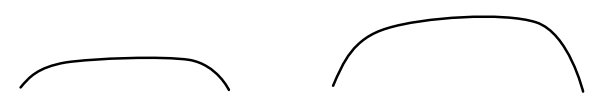

Ex. : $\quad\left(\right.$ la MAjeure parTIe) ${ }_{\mathrm{GA}}$; (BRUmes matiNALes) ${ }_{\mathrm{GA}}$ (Fónagy 1979 : 142).

En revanche, l'A2 ne peut pas être sur la syllabe précédant immédiatement la syllabe

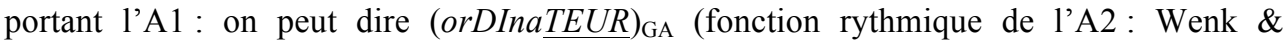
Wioland 1982, Verluyten 1984) ou (ORdinaTEUR $)_{\mathrm{GA}}$ (arc accentuel : Fónagy 1979), mais pas *(ordiNATEUR $)_{\mathrm{GA}}$. Ce principe de non collision accentuelle supprime l'A2 ou le pousse vers le début du GA.

Lorsque le GN est complété à droite par un GAdj ou un GPrép., il y a généralement deux GA et donc deux A1, avec éventuellement des A2 supplémentaires.

Ex. : $\quad(\text { le PEtit jarDIN })_{\mathrm{GA}}(\text { potaGER })_{\mathrm{GA}}$

(le PEtit jarDIN) $)_{\mathrm{GA}}$ (mal enTREteNU) ${ }_{\mathrm{GA}}$

(La MAIson BLANCHe) $)_{\mathrm{GA}}$ (de nos voi $\left.\underline{\text { SINs }}\right)_{\mathrm{GA}}$

Les mots grammaticaux (i.e. les Prép., Dét., auxiliaires, conjonctions...) sont en principe non accentués, comme ici vous, de (mots proclitiques), le et la (Dét.) :

Ex. : $\quad(\text { Vous tourNEZ) })_{\mathrm{GA}}(\text { le } \underline{C O I N})_{\mathrm{GA}}(\text { de la } \underline{R U E} .)_{\mathrm{GA}}$ (Garde 1968 : 94)

Les mots grammaticaux peuvent être accentués seulement quand ils sont en contraste.

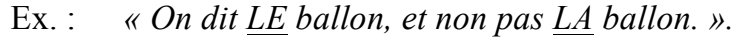

\subsection{L'A1 et l'A2 en turc}

$\mathrm{Au}$ niveau syntaxique, le turc est une langue de structure SOV, dans laquelle les mots modificateurs (par exemple GAdj. ou $\mathrm{N}$ au génitif, désormais $\mathrm{N}$-Gén.) précèdent le $\mathrm{N}$ tête dans le GN. On a donc :

$$
\begin{array}{ll}
{[(\mathrm{GAdj} .)(\mathrm{N}-\mathrm{Gén} .) \mathrm{N}]_{\mathrm{GN}}} & \\
\text { Ex. : } & \text { Klrmizı ev. La maison rouge. (Y1lmaz \& Uras Y1lmaz } 2011: 111) \\
& {[\text { GAdj. }+\mathrm{N}]_{\mathrm{GN}}}
\end{array}
$$

Le GAdj. peut être placé après le $\mathrm{N}$ et en position finale dans la Phrase $(P)$, mais dans ce cas il a la fonction d'un attribut à valeur prédicative, il n'est plus un épithète à valeur qualificative (Uras Y1lmaz, $2004: 35$ ).

Ex. : $\quad$ Ev kırmızı. La maison est rouge. (Y1lmaz \& Uras Y1lmaz $2011: 111$ )

$$
[\mathrm{N}+\text { GAdj. }]_{\mathrm{P}}
$$

Prenons maintenant le cas d'un GN constitué d'un N et d'un N-Gén. :

Ex. : Fransa'nın baskenti. La capitale de la France. (Akınc1 2013-2014 : 26) $[\mathrm{N}-\mathrm{Gén} .+\mathrm{N}]_{\mathrm{GN}}$

Il y a donc des différences avec le français : outre la structure syntaxique SOV en turc ( vs SVO en français) et l'ordre des constituants dans le GN (GAdj. ou N-Gén. avant le N tête en turc vs après le $\mathrm{N}$ tête en français), il n’y a pas de Dét. (défini, possessif...) ni de Prép., mais des Suffixes (Suff.) flexionnels et enclitiques, car c'est une langue agglutinante. On obtient donc de longs mots avec plusieurs Suff. qui sont ajoutés aux racines lexicales et verbales (Akınc1 2013-2014:26).
Ex. : $E v$
maison
Ev-ler maisons
Ev-ler-deki ce qui est présent dans les maisons
Ev-ler-deki-ler ceux qui sont présents dans les maisons $[\mathrm{N}+\text { Suff. }]_{\mathrm{N}}$

Au niveau prosodique, un item lexical et ses Suff. correspondent à un constituant appelé Mot Prosodique $(M P)$, domaine à l'intérieur duquel l'A1 est attribué (Nespor \& Vogel 1986 : 120). Un item lexical est donc toujours associé à un A1 en turc : il s'agit de ce point de vue d'un accent de mot, tandis qu'en français il s'agit d'un accent de groupe.

$[\mathrm{N}+\text { Suff. }]_{\mathrm{N}} \rightarrow[\mathrm{N}+\text { Suff. }]_{\mathrm{MP}}$ 
L'A1 du turc a été beaucoup étudié et discuté. Par défaut, il est final: en cas de suffixation, il se déplace sur la dernière syllabe du dernier suffixe (Van der Hulst \& Van der Weijer 1991, Kornfilt 1997, Lewis 2000, Göksel \& Kerslake 2005, Levi 2005), et délimite ainsi la frontière droite du MP. L'A1 est alors considéré comme non marqué (Levi 2002 : 2).

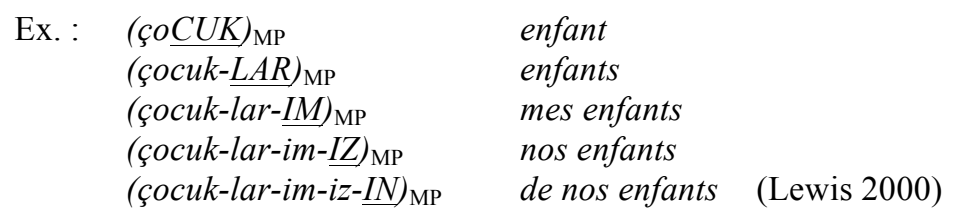

On observe que les Suff. flexionnels et enclitiques sont accentuables en turc, et donc souvent accentués d'après la règle générale d'accentuation finale, alors que les mots grammaticaux proclitiques sont rarement accentués en français, comme nous l'avons vu.

Mais certaines catégories de mots turcs ont leur A1 sur une autre syllabe que la syllabe finale : l'A1 est alors considéré comme marqué (Levi 2002 : 2-3). Il existe ainsi plusieurs sous-systèmes à accent fixe et d'autres à accent libre. Concernant ceux à accent fixe, par exemple les $\mathrm{N}$ Propres $(N P)$ polysyllabiques de lieux, les $\mathrm{N}$ de personnes et les emprunts ne sont jamais oxytoniques : cette catégorie de mots est connue sous l'appellation d'accent Sezer, du nom de l'auteur qui l'a découverte (Sezer 1983). Ces mots gardent leur A1 sur la même syllabe même en cas d'ajout de Suff. (Lewis 2000).

$$
\begin{aligned}
& \text { Ex.: } \quad(A F R I k a)_{\mathrm{MP}} \quad \text { Afrique } \\
& \text { (AFRIka-ll })_{\mathrm{MP}} \quad \text { Africain } \\
& \text { (AFRIka-ll-lar })_{\mathrm{MP}} \quad \text { Africains } \\
& \text { (AFRIka-ll-lar-ln })_{\mathrm{MP}} \text { des Africains (Göksel \& Kerslake 2005) }
\end{aligned}
$$

Un autre cas de sous-système à accent fixe non final est l'A1 placé souvent sur la syllabe initiale dans les Adverbes ( $A d v$.) (Kornfilt 1997, Lewis 2000, Göksel \& Kerslake 2005), etc. Certains Adv. forment ainsi des paires minimales avec d'autres items lexicaux (N, Adj., Verbes) dans lesquels l'A1 joue un rôle distinctif, ce qui confirme que 1'A1 turc est alors un accent lexical:

$$
\begin{array}{ll}
\text { Ex.: } & \text { ÇOkluk (souvent) } \sim \text { çoKLUK } \\
& \underline{\text { YALniz (meultitude) }}
\end{array}
$$

$\mathrm{Au}$ niveau de ses traits acoustiques, l'A1 final en turc est réalisé par un accent mélodique Haut (noté $\mathrm{H}^{*}$ ) ou Bas (noté $\mathrm{B}^{*}$ ), en fonction de l'intonation de l'énoncé (Underhill 1976, Konrot 1981, Lewis 1967/2000, Göksel \& Kerslake 2005, Kawaguchi \& al. 2005, Levi 2002, 2005). De ce point de vue, l'A1 turc fonctionne comme l'A1 français.

L'A2 turc est facultatif, avec moins de hauteur mélodique que 1'A1, mais plus qu'une syllabe inaccentuée (Kornfilt 1997 : 503-504). Il peut se trouver dans un groupe ou un mot composé, où le mot modificateur porte l'A1 de groupe et où le mot principal porte l'A2 :

Ex.: $\quad \underline{B A S ̧}($ tête $)+b a \underline{K A N}($ ministre $)=>(\underline{B A S ̧ b a K A N})_{\mathrm{MP}}$ (Premier Ministre $)$

Dans les mots dont 1'A1 est non final et/ou ayant plusieurs Suff., on peut trouver un A2 en position finale de mot, surtout s'il est assez éloigné de l'A1 (Van der Hulst \& Van der Weijer 1991 : 10) :

Ex. : (loKANta-lar-ım-lz-DAN) $)_{\mathrm{MP}}$ (de nos restaurants) restaurant-P1.- ${ }^{\text {ère }}$ Pers.P1.-de

Voici maintenant une synthèse des points communs et des différences principales entre l'A1 et l'A2 en français et en turc. À partir de cette analyse contrastive, on peut aussi comparer le degré de la Marque des accents des deux langues, dans le cadre de l'HDM (Eckman 1977/2008). Le domaine d'application des deux accents est le GA en français vs le MP en turc. L'A1 est final en français ; en turc, l'A1 régulier est final, tandis que l'A1 lexical est libre et non final. L'A1 français et l'A1 régulier turc ont une fonction démarcative (ils indiquent respectivement la fin des GA et des MP) ; tandis que l'A1 lexical turc a une fonction distinctive. On peut donc avancer que l'A1 turc semble au moins aussi 
marqué que l'A1 français, car il obéit à plus de règles de placement différentes (selon les catégories grammaticales des mots et des Suff.) et car il a deux fonctions, alors que l'A1 français est toujours final et n'a qu'une fonction ; cependant, ce dernier est complexe aussi car il peut porter sur un GA plus ou moins long. L'A2 français est surtout initial, mais pas toujours, et forme avec l'A1 un arc accentuel (étendu), avec une fonction rythmique et démarcative (il indique le début des GA), alors que 1'A2 turc est final, avec une fonction démarcative seulement (il indique la fin des MP dans les mots dont l'A1 est lexical) : la place de 1'A2 semble donc plus marquée en français qu'en turc. Les deux accents sont réalisés en français avec deux paramètres acoustiques ( $\mathrm{F}_{0}$ et durée) vs avec un seul paramètre $\left(\mathrm{F}_{0}\right)$ en turc, donc ils sont moins marqués en turc du point de vue acoustique.

\section{Hypothèses}

Étant donné les convergences et divergences des systèmes accentuels du turc et du français, à la fois aux niveaux phonologique et phonétique, et les différences de Marque entre les A1 et A2 des deux langues (HDM, Eckman 1977/2008), dans quelle mesure les locuteurs adultes plurilingues de Li turque transfèrent-ils les particularités de l'A1 et de l'A2 turcs dans le GN en français La, et de quels types sont ces transferts?

L'A1 turc étant au moins aussi marqué, donc aussi complexe que l'A1 français du point de vue de deux critères sur quatre (son placement et ses fonctions), nous estimons que les locuteurs de turc Li n'auront pas de problème de surdité accentuelle (Dupoux \& al. 1997/2010) : ils devraient repérer les différences de l'A1 français et les acquérir assez facilement, donc faire peu, voire pas d'erreurs accentuelles au niveau phonologique dans le GN en français. On s'attend à un transfert positif de l'A1 régulier final du turc sur les fins de GA en français, sans transfert négatif de l'A1 lexical turc. En revanche, l'A2 français étant non final et plus marqué que 1'A2 turc (concernant à la fois son placement, ses fonctions et ses paramètres), ne pourrait-il pas être interprété par les locuteurs turcs comme un A1 lexical ? Par ailleurs, l'A1 et l'A2 étant plus marqués en français qu'en turc sur le plan acoustique (deux paramètres $v s$ un seul), on peut s'attendre à des variantes au niveau phonétique : les locuteurs n'auront-ils pas des difficultés à gérer les proportions de l'allongement (paramètre non utilisé en turc), mais aussi des variations de $\mathrm{F}_{0}$ en français ? Ces difficultés et/ou variantes phonétiques pourraient apparaître aussi sur les Dét. et Prép. en français, étant donné qu'en turc ce sont des Suff. flexionnels et enclitiques souvent accentués, d'après la règle générale d'accentuation finale.

\section{Méthodologie}

Afin d'étudier les réalisations accentuelles des turcophones en français, nous nous sommes basés sur une démarche expérimentale et une analyse acoustique.

\subsection{Corpus}

Un corpus de 24 phrases déclaratives simples de structure SVO a été constitué, dans lequel on a fait varier de façon systématique et contrôlée la structure syntaxique et prosodique des GA (nombre de syllabes, structure syntaxique et nombre d'accents) et leur place dans les phrases. Les phrases $\mathrm{n}^{\circ} 17$ et $\mathrm{n}^{\circ} 18$ ci-dessous sont représentatives de ce corpus. Le $\mathrm{N}$ cible, i.e. celui dont l'accentuation a été plus particulièrement étudiée (ici ciboulette), est toujours le N tête du GN Sujet : selon si son Dét. et lui sont les deux seuls constituants du Sujet ou s'il est complété à sa droite par un GPrép., ce $\mathrm{N}$ se trouve respectivement à une coupe syntaxique majeure, notée // (phrase $\left.{ }^{\circ} 17\right)$ ou mineure, notée / $\left(\right.$ phrase $\left.^{\circ}{ }^{\circ} 18\right)$. 


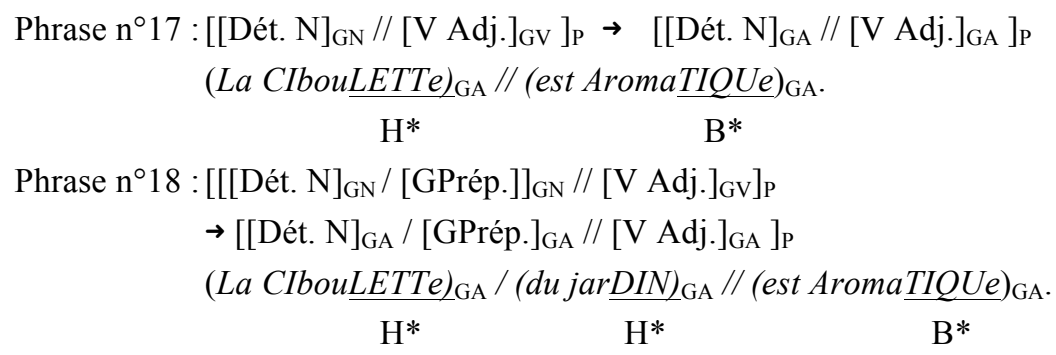

Dans ce corpus, les mots grammaticaux font partie des syllabes inaccentuées, donc réalisées en français $\mathrm{Li}$ avec une durée courte et un minimum de $\mathrm{F}_{0}$ (Rossi 1980/1999, Post 2000, Di Cristo 1998/2016).

\subsection{Locuteurs}

Les trois locuteurs (Loc. 1, Loc. 2, Loc. 3) sont des femmes de 22 à 28 ans, parlent le turc de référence de Turquie et étudient dans les universités turques et françaises. Le français est leur L3, car elles ont étudié l'anglais auparavant. Deux d'entre elles (Loc. 2 et 3) ont étudié le français seulement 10 mois, depuis leur arrivée en France et sont de niveau A1 du point de vue du Cadre Européen Commun de Référence pour les Langues (CECRL). En revanche, la Loc. 1 parle une deuxième Li, le kurde (bilinguisme précoce), et comptabilise sept ans d'apprentissage du français : elle est de niveau B2 à l'écrit et C1 à l'oral (CECRL).

\subsection{Tâche}

La tâche choisie est la répétition : les productions sonores en français Li d'un locuteur de référence ( $L o c$. de réf. ou $L R$ ) faisant une lecture oralisée du corpus ont été enregistrées, puis les locutrices ont écouté et répété ses productions en l'imitant le mieux possible sur le plan prosodique. Le LR est David Le Gac lui-même, l'un des deux auteurs de cet article, car sa production orale a été identifiée comme du français de référence lors de tests de perception (Detey \& Le Gac 2010). La tâche de répétition permet d'aider les locuteurs à mémoriser la prosodie des énoncés et leur fait travailler à la fois la perception et la production. Comparée à une tâche de lecture oralisée, elle permet aussi de neutraliser les difficultés liées à l'input visuel de l'écrit (l'orthographe de la langue française) et de donner la même référence à tous les locuteurs. Les phrases du corpus ont été isolées, mélangées et tirées au sort pour le LR comme pour les locutrices turques afin d'éviter l'effet de liste et d'obtenir une prononciation plus naturelle. Chacune a réalisé trois productions sonores du corpus entier (enregistrements E1,E2, E3), afin d'observer une éventuelle évolution.

\subsection{Analyse des données}

Les données ont été enregistrées et annotées avec Praat, logiciel d'analyse acoustique de la parole (Boersma \& Weenink 2012), en relevant automatiquement pour chaque syllabe la durée en millisecondes $(\mathrm{ms})$ et 10 points de mesure de $\mathrm{F}_{0}$ en Hertz $(\mathrm{Hz})$ répartis de façon homogène (tous les $10 \mathrm{e}$ de seconde). Cela permet d'obtenir une courbe mélodique proche de la courbe de $\mathrm{F}_{0}$ réelle, et surtout de normaliser la $\mathrm{F}_{0}$ sur l'axe temporel et de pouvoir ainsi comparer les locuteurs sur cet axe. Dans une même optique, les données de $\mathrm{F}_{0}$ en $\mathrm{Hz}$ ont été converties en demi-tons, afin de pouvoir comparer les locutrices au LR, qui est un homme. La Valeur $(\mathrm{Val})$ de Référence $(R E F)$ prise a été $100 \mathrm{~Hz}$, car cela correspond au registre bas $\mathrm{du}$ LR. La formule de calcul utilisée est la fonction logarithmique suivante : $12 \mathrm{x}$ $\log _{2}(\mathrm{Val} / \mathrm{REF})$ (t'Hart \& al. 1990). Les productions des locutrices ont été comparées à celle du LR. 
Sur le plan phonologique, les données ont été interprétées dans le cadre de la phonologie métrique et autosegmentale (Pierrehumbert \& Beckman 1980/1988, Ladd 1996/2008), cadre de référence dans les études actuelles en prosodie, et dans lequel le français et le turc ont déjà été décrits, ce qui facilite la comparaison (pour le français : Jun \& Fougeron 1995/2000, Post 2000, Gussenhoven 2004, Di Cristo 1998/2016 ; pour le turc : Ladd 1996/2008).

\section{Résultats}

Les graphiques ci-dessous sont des exemples très représentatifs des réalisations les plus courantes des trois locutrices turques.

\subsection{Gestion phonologique des accents (place et paramètres acoustiques utilisés)}

On voit sur les figures 1 et 2 que l'A1 et l'A2 sont bien placés par la Loc. 1 dans l'énoncé 18 : La ciboulette du jardin est aromatique. On a en effet la réalisation suivante :

$$
(\text { La CIbouLETTe })_{\mathrm{GA}}(d u \operatorname{jarDIN})_{\mathrm{GA}}(\text { est AromaTIQUe })_{\mathrm{GA}} \text {. }
$$

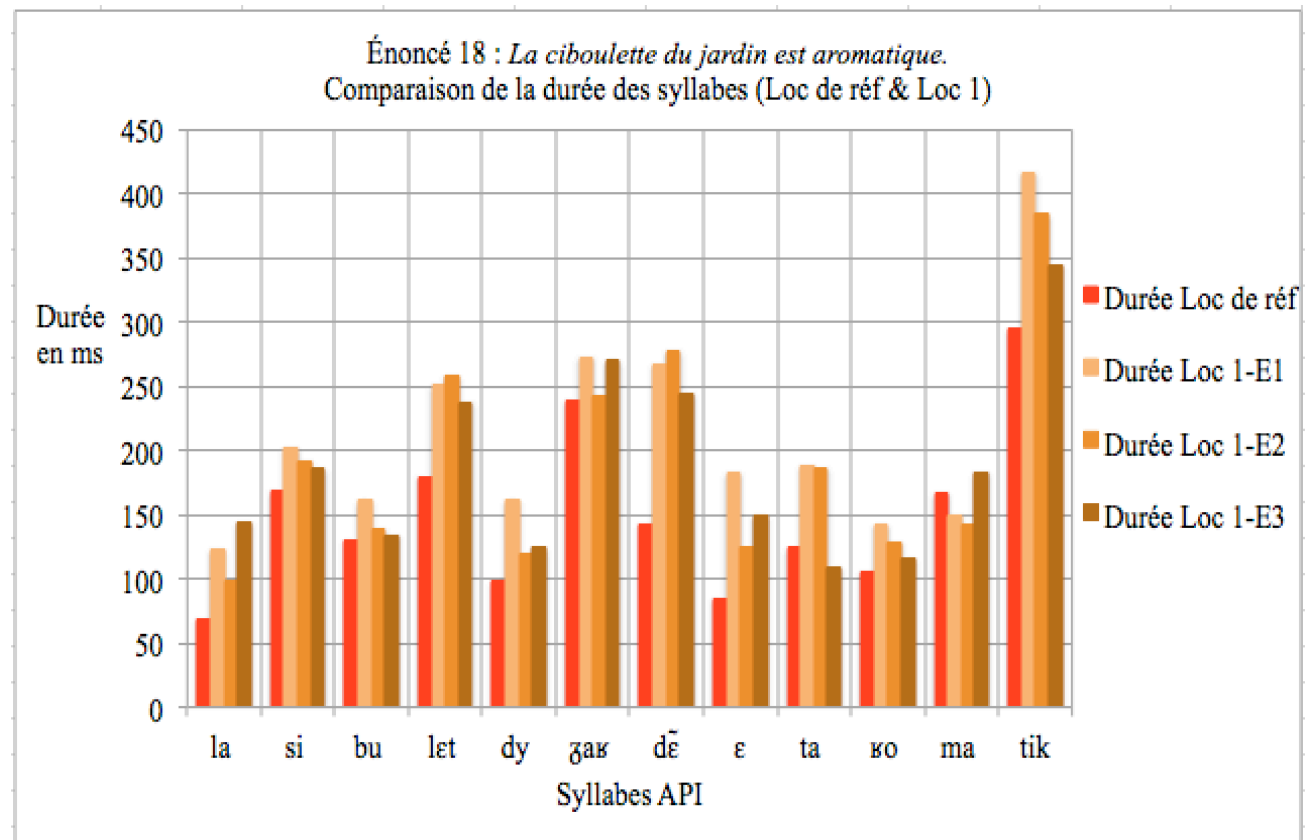

Figure 1. Comparaison de la durée des syllabes dans l'énoncé 18 (LR \& Loc 1) : La ciboulette du jardin est aromatique.

L'A1 porte bien sur la syllabe finale des trois GA de l'énoncé, avec les deux paramètres acoustiques attendus, i.e. un allongement et $\mathrm{F}_{0}$ : une hausse mélodique notée $\mathrm{H}^{*}$ sur les deux premiers A1 pour indiquer la présence d'une suite dans l'énoncé (intonation continuative) et une baisse mélodique notée B* sur l'A1 final de l'énoncé déclaratif. Quant à l'A2, il porte bien sur la syllabe initiale du premier mot lexical d'au moins trois syllabes dans chaque GA : CIbouLETTe, AromaTIQUe. La gestion phonologique des accents est donc correcte. 


\subsection{Réalisation phonétique des accents}

$\mathrm{Au}$ niveau de leur réalisation phonétique, il $\mathrm{y}$ a des variantes : on observe plus d'allongement que chez le LR, surtout sur les syllabes portant l'A1 (figure 1), mais en revanche des hausses de $\mathrm{F}_{0}$ moins élevées concernant les pics accentuels et un registre d'ensemble moins important (figure 2). On observe aussi que l'alignement temporel du pic mélodique sur la syllabe portant l'A1 du mot jarDIN est un peu plus tardif dans E1 et E2 que celui du LR (figure 2). Ces différents phénomènes prosodiques sont récurrents.

Les figures 3 et 4 ci-dessous montrent encore plus nettement certains phénomènes. La figure 3 montre la réalisation de la phrase $n^{\circ} 22$ par la Loc. 2. On constate que la gestion phonologique des accents est toujours correcte car on a la réalisation suivante :

$$
(\text { Le MÉloMANe })_{\mathrm{GA}}\left(d^{\prime} \text { 'à côTÉ) } \frac{\mathrm{H}_{\mathrm{GA}}}{\mathrm{H}^{*}}\left(\text { aime } m a \frac{m u S I Q U e}{\mathrm{BA}}\right)_{\mathrm{BA}}\right. \text {. }
$$

On peut faire la même observation avec la figure 4 (phrase $n^{\circ} 17$ prononcée par la Loc. $3)$, où l'on a cette réalisation :

$$
(\text { La CIbouLETTe })_{\mathrm{GA}}(\text { est AromaTIQUe })_{\mathrm{GA}} \text {. }
$$

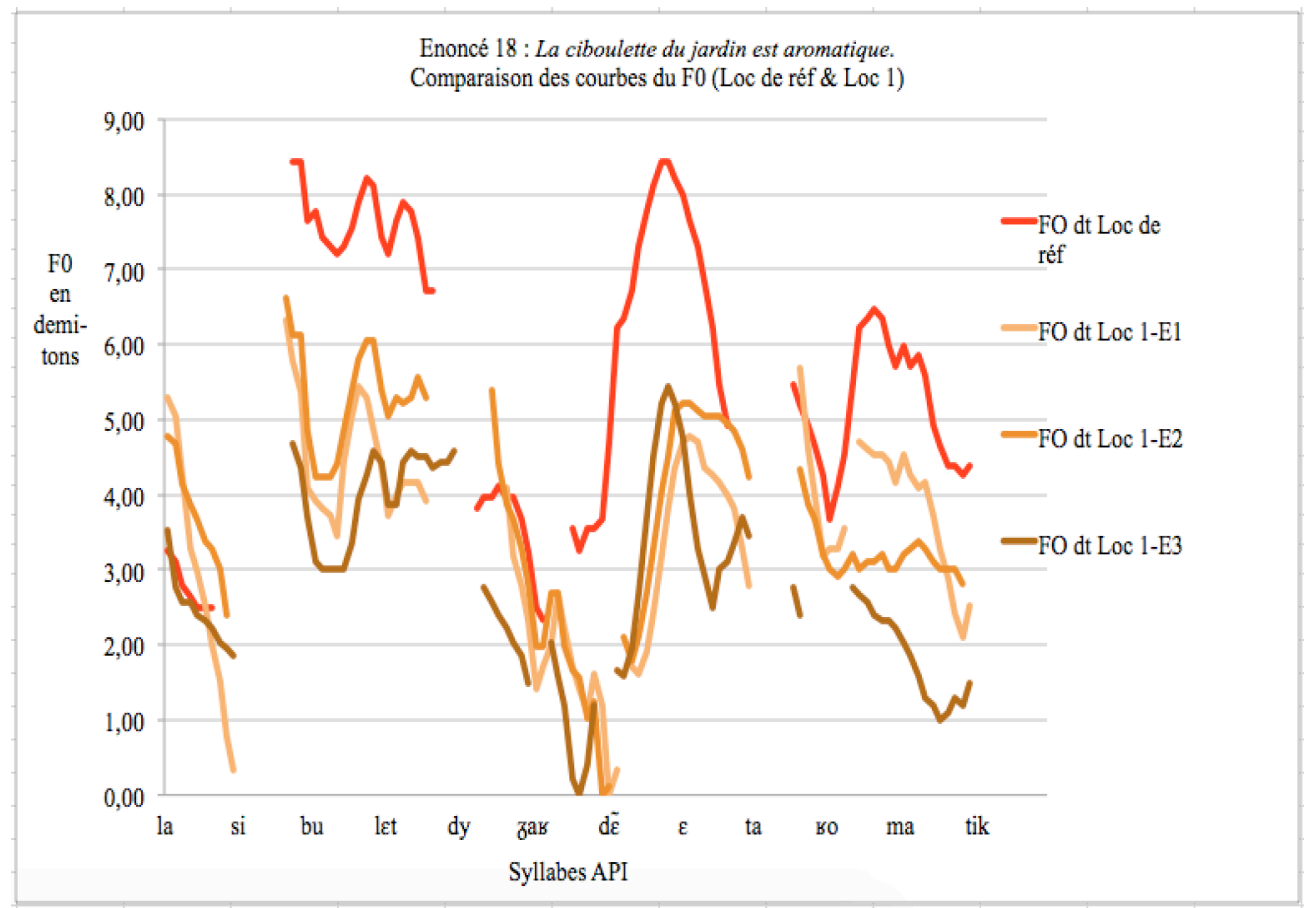

Figure 2. Comparaison des courbes du $\mathrm{F}_{0}$ dans l'énoncé 18 (LR \& Loc 1) : La ciboulette du jardin est aromatique.

En revanche, ces trois figures montrent nettement les deux phénomènes prosodiques les plus significatifs dans les productions des locutrices turques en français : une gamme de fréquences plus étroite et surtout le fait que le niveau mélodique d'ensemble est plus bas que celui du LR. 


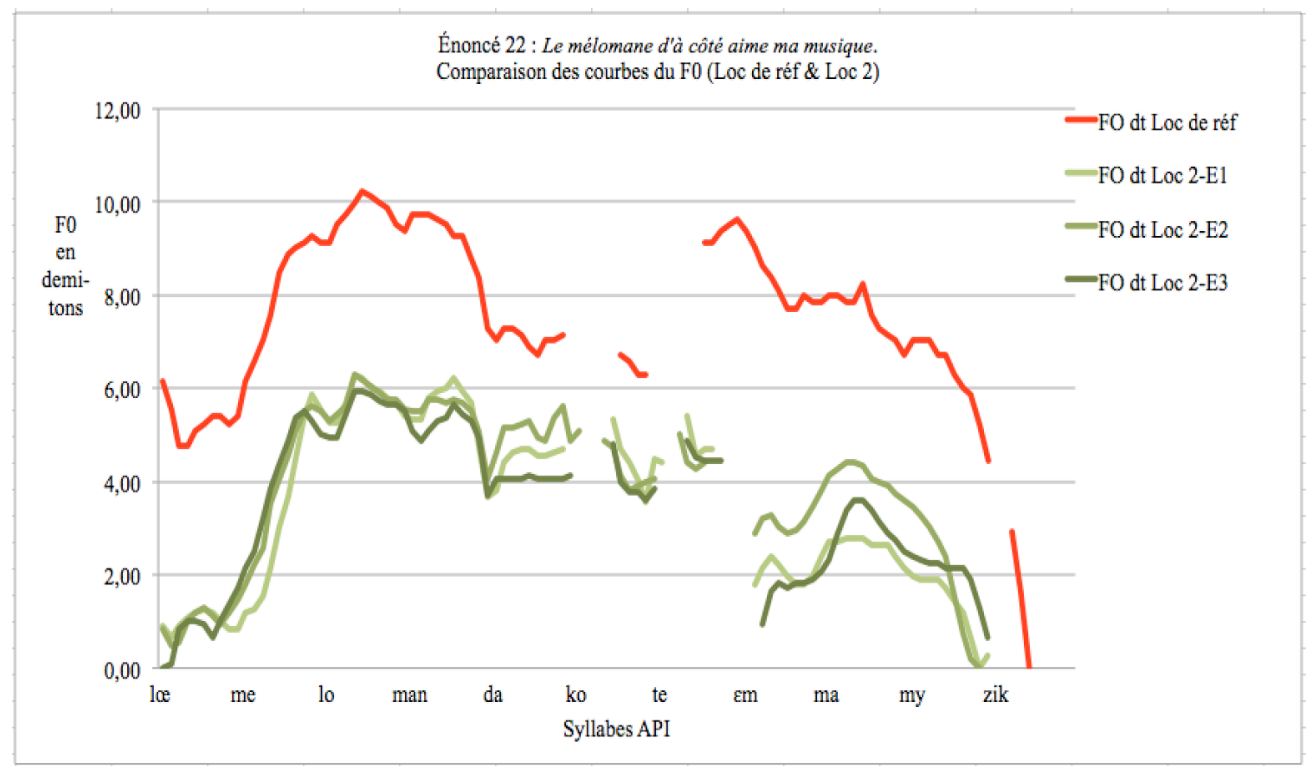

Figure 3. Comparaison des courbes du $\mathrm{F}_{0}$ dans l'énoncé 22 (LR \& Loc 2) : Le mélomane d'à côté aime ma musique.

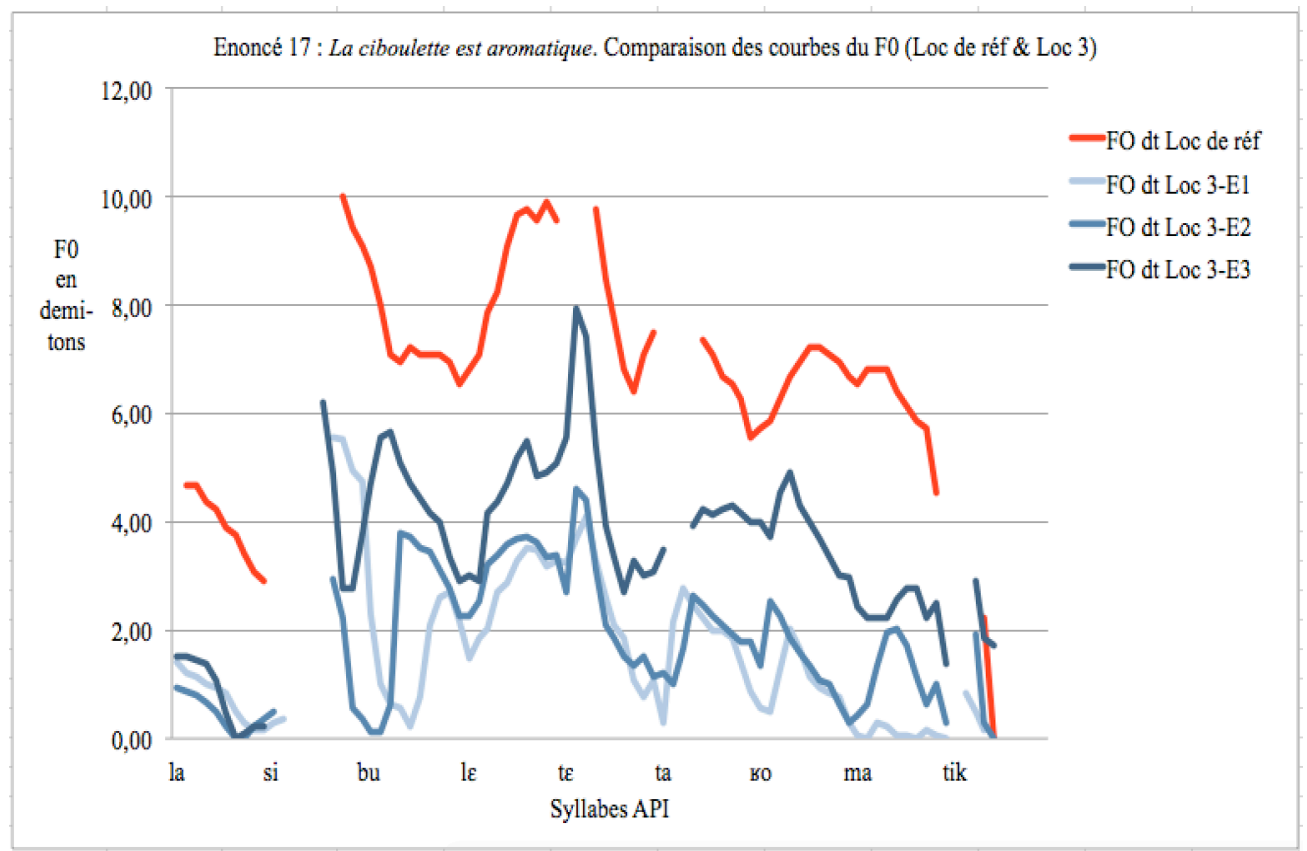

Figure 4. Comparaison des courbes du $\mathrm{F}_{0}$ dans l'énoncé 17 (LR \& Loc 3) : La ciboulette est aromatique.

Cela dit, ce double phénomène (gamme plus étroite et niveau mélodique global plus bas en français La) n'a été constaté que lors de l'analyse des données et de la réalisation des graphiques comparatifs entre chaque locutrice turque et le LR, car cela n'était pas frappant à la simple perception. Il semble donc que ces phénomènes entrent dans le spectre des variations possibles du français parlé, au même titre que la variabilité inter-locuteurs en français $\mathrm{Li}$. 
Enfin, on remarque dans la figure 4 que la deuxième syllabe du mot ciboulette, [bu], est réalisée avec une hausse mélodique de plus en plus marquée et de plus en plus précoce par la Loc. 3, alors qu'il s'agit d'une syllabe inaccentuée : cela montre sa difficulté à gérer les alignements temporels et les variations de $\mathrm{F}_{0}$, y compris sur les syllabes inaccentuées. C'est également le cas de certains Dét. et Prép. dans les productions des trois locutrices.

\section{Discussion}

Les résultats confirment nos hypothèses et nous amènent à discuter, dans le domaine de la prosodie, certaines approches sur l'acquisition de la prononciation d'une La. Les locutrices turques de français La ne font pas d'erreurs au niveau phonologique : elles gèrent bien la distribution de 1'A1 et de l'A2 (domaine et place), mais aussi leur réalisation acoustique, avec les deux paramètres pertinents ( $\mathrm{F}_{0}$ et durée). La structuration prosodique des énoncés (phrasing) est donc correctement réalisée et les deux accents ont bien leur fonction démarcative en français : l'A1 en fin de GA et l'A2 en début de GA. Comme d'autres études auparavant, nos premiers résultats paraissent donc aller à l'encontre de la version prédictive de l'HAC (Lado 1957), qui affirme qu'un système linguistique différent est difficile à acquérir. Nous avons vu aussi que les ressemblances entre les deux systèmes accentuels (en particulier entre l'A1 français systématiquement final et l'A1 turc régulier final) n'ont pas posé de problème non plus aux locutrices : cela amène donc aussi à s'interroger sur la version modérée de l'HAC (Wardhaugh 1970, Brown 1987, Flege 1992), qui estime quant à elle que ce sont les ressemblances entre les deux systèmes linguistiques qui posent problème aux locuteurs.

Nos résultats confirment plutôt les prédictions de l'HDM (Eckman 1977/2008), tout comme d'autres études précédentes. L'A1 turc étant au moins aussi marqué que l'A1 français, les locutrices turques n'ont pas de problème de surdité accentuelle (Dupoux \& al. 1997/2010), donc pas de difficulté dans la gestion phonologique de l'A1 en français : il y a bien un transfert positif de l'A1 régulier final du turc à la fin des GA en français, sans transfert négatif de 1'A1 lexical du turc, et l'A2 français n'est pas interprété comme un A1 lexical. Notre étude va donc dans le même sens que celle de Rasier \& Hiligsmann (2007) : leurs locuteurs de néerlandais $\mathrm{Li}$ acquièrent plus facilement l'accentuation en français que leurs locuteurs de français Li n'acquièrent celle du néerlandais, car celle du néerlandais est plus marquée que celle du français.

Par ailleurs, l'A1 et l'A2 étant plus marqués en français qu'en turc sur le plan acoustique (deux paramètres vs un seul), nos hypothèses au niveau phonétique se confirment également : les locutrices turques ont des difficultés à gérer les proportions de l'allongement (paramètre non utilisé en turc), mais aussi des variations de $\mathrm{F}_{0}$ en français. En effet, la hiérarchie des patrons intonatifs continuatifs (mineur vs majeur) est très importante en français, proportionnellement à la hiérarchie des coupes syntaxiques (mineure vs majeure) (Rossi 1999, Post 2000, Di Cristo 1998/2016). Ces variantes phonétiques des locutrices turques semblent toutefois faire partie de la variabilité des locuteurs de français $\mathrm{Li}$ : une étude plus large et plus approfondie, ainsi que des tests de perception, permettraient de voir si les locuteurs de français Li confirment cette impression. Enfin, comme nous l'avions supposé, des difficultés et/ou variantes phonétiques de $\mathrm{F}_{0}$ et de durée apparaissent sur certaines syllabes inaccentuées de certains mots lexicaux, ainsi que sur certains Dét. et Prép. en français La, peut-être parce que ces mots grammaticaux sont des Suff. de construction et de déclinaison accentuables en turc.

\section{Conclusion}


Les résultats montrent donc que les locuteurs turcs, au niveau phonologique, réalisent bien l'A1 en fin de GA en français $\mathrm{La}$, avec les paramètres acoustiques attendus $\left(\mathrm{F}_{0}\right.$ et durée $)$ : il y a un transfert positif de l'A1 régulier turc, pas de transfert négatif de l'A1 lexical turc, et l'A2 français initial n'est pas interprété comme un A1 non final, il est réalisé le plus souvent comme en français Li. Ces résultats vont à l'encontre des versions prédictive (Lado 1957 ) et modérée (Wardhaugh 1970, Brown 1987, Flege 1992) de l'HAC ; ils vont plutôt dans le sens de l'HDM (Eckman 1977/2008) et de l'Hypothèse de la surdité accentuelle (Dupoux \& al. 1997/2010). En effet, l'A1 turc étant au moins aussi marqué, i.e. aussi complexe dans sa gestion phonologique que l'A1 français, les locuteurs turcs ne sont pas sourds aux différences de l'A1 et de l'A2 français. Notre étude va donc dans le même sens que celle de Rasier et Hiligsmann (2007).

$\mathrm{Au}$ niveau phonétique, les difficultés et variantes prévues se confirment : les locuteurs turcs ont du mal à gérer en français les proportions des allongements et surtout des variations de $\mathrm{F}_{0}$, en fonction notamment de l'importance des frontières syntaxiques et prosodiques (phrasing). La durée plus importante des syllabes portant l'A1, par rapport au LR, confirme certains résultats d'autres études sur le français La (Schwab 2012). Par ailleurs, ces allongements plus importants, tout comme les alignements temporels plus tardifs des pics accentuels, ainsi que le niveau mélodique global plus bas et la gamme de fréquences plus étroite, ne semblent pas avoir d'impact sur la compréhensibilité des locuteurs turcs en français La et semblent entrer dans la variabilité des locuteurs de français Li. Ces différences phonétiques vont dans le même sens que d'autres recherches (Mennen 2007, Mennen \& al. 2012).

En revanche, dans notre étude, les locuteurs réalisent une $\mathrm{F}_{0}$ moins haute pour les pics accentuels comme pour le niveau d'ensemble, contrairement à l'étude de Schwab (2012). D'autres investigations seraient donc nécessaires avec les mêmes locuteurs, pour voir s'ils s'expriment aussi avec un registre mélodique bas quand ils parlent en turc Li, en anglais et en français : en effet, nos résultats rejoignent pour l'instant ceux d'autres études (Campione et Veronis 1998, Mennen \& al. 2012, Andreeva \& al. 2014), qui montrent que les locuteurs bi- ou plurilingues n'utilisent pas le même niveau ni la même gamme de $\mathrm{F}_{0}$ selon la langue qu'ils parlent. Si ce phénomène prosodique se confirmait, cela aurait une importance et des applications concrètes en vue de l'identification des locuteurs, dans des domaines tels que la reconnaissance vocale, la synthèse de la parole, la phonétique judiciaire...

\section{Références bibliographiques}

Akıncı, M.-A. (2013-2014). Eléments de cours de turc, Expérience d'Apprentissage d'une Langue Entièrement Nouvelle. Cours d'enseignement à distance, Licence 3 de Sciences du Langage FLE, Université de Rouen Normandie.

Andreeva, B., Demenko, G., Wolska, M., Möbius, B., Zimmerer, F., Jügler, J., Jastrzebska, M. et Trouvain, J. (2014). Comparison of Pitch Range and Pitch Variation in Slavic and Germanic Languages. Proceedings of Speech Prosody, 7, 776-780.

Boersma, P. et Weenink, D. (2012). Praat : Doing Phonetics by computer (version 5.3.56). Logiciel d'analyse acoustique de la parole. Institut de Sciences Phonétiques, Université d'Amsterdam, disponible et téléchargeable en ligne à l'adresse suivante : http://www.fon.hum.uva.nl/praat/

Brown, H. D. (1987). Principles of Language Learning and Teaching. New Jersey: Prentice Hall (2 ${ }^{\text {nd }}$ ed.).

Campione, E. et Véronis, J. (1998). A statistical study of pitch target points in five languages. 5th International Conference on Spoken Language Processing (ICSLP'98), Sidney, 1391-1394.

Corder, S. (1967). The significance of learners' errors. International Review of Applied Linguistics in Language Teaching, 5 (4), 161-170.

Corder, S. (1974). Indiosyncratic errors and Error Analysis. International Review of Applied Linguistics, 9 (2), 147-159.

Derwing, T. M. et Munro, M. J. (2009). Putting accent in its place: Rethinking obstacles to communication. Language Teaching, 42 (4), 476-490. 
Detey, S. et Le Gac, D. (2010). Le français de référence : quels locuteurs ? Detey, S., Durand, J., Laks, B. et Lyche, C. (2010). Les variétés du français parlé dans l'espace francophone. Paris : Ophrys, 167-180.

Di Cristo, A. (1998). Intonation in French. Hirst, D. et Di Cristo, A. (eds). Intonation Systems: A Survey of Twenty Languages. Cambridge: Cambridge University Press, 195-218.

Di Cristo, A. (2016). Les musiques du français parlé. Berlin/Boston: de Gruyter, Coll. Études de Linguistique Française.

Duběda, T. (2012). L'accent initial dans le français des apprenants tchèques : aspects distributionnels et intonatifs. Etudes de prosodie contrastive : le cas du français et du tchèque. Université Charles de Prague : Karolinum, 86-97.

Dupoux, E., Pallier, C., Sebastián-Gallés, N. et Mehler, J. (1997). A destressing 'deafness' in French? Journal of Memory and Language, 36 (3), 406-421.

Dupoux, E., Peperkamp, S. et Sebastián-Gallés, N. (2001). A robust method to study stress 'deafness'. Journal of the Acoustical Society of America, 110 (3), 1606-1618.

Dupoux, E., Peperkamp, S. et Sebastián-Gallés, N. (2010). Limits of/on bilingualism revisited: Stress 'deafness' in simultaneous French-Spanish bilinguals. Cognition, 114 (2), 266-275.

Dupoux, E., Sebastián-Gallés, N., Navarrete, E. et Peperkamp, S. (2008). Persistent stress 'deafness': The case of French learners of Spanish. Cognition, 106, 682-706.

Eckman, F. R. (1977). Markedness and the Contrastive Analysis Hypothesis. Language Learning, 27, 315-330.

Eckman, F. R. (2008). Typological Markedness and Second Language Phonology. Edwards, J. G. H. et Zampini, M. L. (eds) (2008). Phonology and Second Language Acquisition. Amsterdam / Philadelphia: John Benjamins Publishing Company, 95-115.

Field, J. (2005). Intelligibility and the listener: The role of lexical stress. TESOL Quarterly, 39, 399423.

Flege, J. E. (1992). Speech Learning in a Second Language. Ferguson, C., Menn, L. et StoelGammon, C. (eds). Phonological Development: Models, Research and Implications. Timonium, MD: York Press, 565-604.

Fónagy, I. (1979). L'accent français: accent probabilitaire (Dynamique d'un changement prosodique). Fónagy, I. et Léon, P. (Dir.). L'accent en français contemporain. Studia Phonetica, 15, Paris : Didier, 123-233.

Garde, P. (1968). L'accent. Paris : PUF.

Göksel, A. et Kerslake, C. (2005). Turkish: a comprehensive grammar. London \& New York: Routledge.

Gussenhoven, C. (2004). The Phonology of Tone and Intonation. Cambridge: Cambridge University Press.

Hahn, L. D. (2004). Primary stress and intelligibility: Research to motivate the teaching of suprasegmentals. TESOL Quarterly, 38, 201-223.

Horgues, C. (2010). Prosodie de l'accent français en anglais et perception par des auditeurs anglophones. Thèse de Doctorat, Université de Paris Diderot Paris 7.

Jenkins, J. (2002). A sociallinguistically based, empirically researched pronunciation syllabus for English as an international language. Applied Linguistics, 23, 83-103.

Jun, S.-A. et Fougeron, C. (1995). The accentual phrase and the prosodic structure of French. Proceedings International Congress of the Phonetic Sciences, 13 (2), 722-725.

Jun, S.-A. et Fougeron, C. (1998/2000). A phonological model of French intonation. Botinis, A. (ed.) Intonation: Models, analysis and applications. Cambridge: Cambridge University Press, 209-242.

Kawaguchi, Y., Yılmaz, S. et Uras Yilmaz, A. (2005). Intonation Patterns of Turkish Interrogatives. Linguistic Informatics IV, Prosody and Sentence Structures, 21st Century COE: Center of UsageBased Linguistic Informatics, Graduate School of Area and Culture Studies, Tokyo University of Foreign Studies.

Kijak, A. (2009). How stressful is L2 stress? A cross-linguistic study of L2 perception and production of metrical systems. Ph. D. Thesis, Graduate School of Netherlands.

Konrot, A. K. (1981). Towards Understanding Turkish Stress: an acoustic and perceptual study. PH. D. Thesis, University of Essex.

Kornfilt, J. (1997). Turkish (Descriptive grammars). London \& New York: Routledge, Series Editor: Bernard Comrie, University of Southern California.

Ladd, D.R. (1996/2008). Intonational Phonology. Cambridge: Cambridge University Press, $2^{\text {nd }}$ ed. 
Lado, R. (1957). Linguistics Across Cultures: Applied Linguistics for Language Teachers. Ann Arbor, Michigan: University of Michigan Press.

Levi, S. V. (2002). Intonation in Turkish: the realization of noun compounds and genitive possessive NPs. UW ms, Washington University.

Levi, S. V. (2005). Acoustic correlates of lexical accent in Turkish. Journal of the International Phonetic Association, 35/1, 73-97.

Lewis, G. L. (1967/2000). Turkish grammar. Oxford - New York: Oxford University Press.

Mennen, I. (2007). Phonological and phonetic influences in non-native intonation. Gut, U. et Trouvain, J. (éds). Non-Native Prosody, Phonetic Description and Teaching Practice. Berlin : Mouton de Gruyter, 53-76.

Mennen, I., Schaeffler, F. et Docherty, G. (2012). Cross-language differences in fundamental frequency range: A comparison of English \& German. Journal of the Acoustical Society of America, 131 (3), 2249-2260.

Nespor, M. et Vogel, I. (1986). Prosodic phonology. Dordrecht: Foris.

Nguyen, T. \& Ingram, J. (2005). Vietnamese acquisition of English word stress. TESOL Quarterly, 39 (2), 309-319.

Pierrehumbert, J. B. (1980). The Phonology and Phonetics of English Intonation. PhD Thesis, Massachusetts Institute of Technology.

Pierrehumbert, J. B. et Beckman, M. (1988). Japanese tone structure. Cambridge, Massachusetts: Massachusetts Institute of Technology Press.

Post, B. (2000). Tonal and phrasal structures in French intonation. The Hague: Holland Academic Graphics.

Rasier, L. et Hiligsmann, Ph. (2007). Prosodic Transfer from L1 to L2. Theoretical and methodological issues. Nouveaux Cahiers de Linguistique Française, 28, 41-66.

Rossi, M. (1980). Le français, langue sans accent? Fónagy, I. et Léon, P. R. L'accent en français contemporain. Studia Phonetica, 15, Montréal-Paris-Bruxelles : Didier, 13-51.

Rossi, M. (1999). L'intonation, le système du français: description et modélisation. Paris : Ophrys.

Santiago, F. (2016). La prosodie des syntagmes intonatifs en français L2: une étude perceptive. Langages, 202 (2), 13-34.

Schwab, S. (2012). Do native Spanish speakers transfer accentual properties from Spanish to French L2? Proceedings of Speech Prosody, 6, 330-333.

Selinker, L. (1972). Interlanguage. International Review of Applied Linguistics in Language Teaching, 10 (3), 209-231.

Sezer, E. (1983). On non-final stress in Turkish. Journal of Turkish Studies, 5, 61-69.

T' Hart, J., Collier, R. et Cohen, A. (1990). A perceptual study of intonation: an experimental phonetic approach to speech melody. Cambridge studies in speech science and communication, Cambridge: Cambridge University Press.

Underhill, R. (1976). Turkish Grammar, Cambridge, MA: MIT Press.

Vaissière, J. (1991). Rhythm, accentuation and final lengthening in French. Carlson, R., Nord, L. et Sundberg, J. (eds). Proceedings of the 1990 Wenner-Gren Center Conference on Music, Language, Speech and Brain. New York: Macmillan, 108-120.

Van der Hulst, H. (2012). Deconstructing stress. Lingua 122, 1494-1521.

Van der Hulst, H. et Van der Weijer, J. (1991). Topics in Turkish Phonology. Turkish Linguistics Today, 11-59.

Verluyten, P. (1984). Phonetic reality of linguistic structures: the case of (secondary) stress in French. Proceedings of ICPhS 10, 522-526.

Wardhaugh, R. (1970). The Contrastive Analysis Hypothesis. TESOL Quarterly 4.

Wenk, B. J. et Wioland, F. (1982). Is French really syllable-timed? Journal of Phonetics, 10 (2), 193216.

Yılmaz, S. (2004). Organisation discursive et intonative du français parlé par un locuteur turc. Comparaison avec la production d'un locuteur français. Francophonie en Turquie, dans les pays balkaniques et de l'Europe orientale. Actes du Colloque International Pluridisciplinaire, Les Cahiers du Bosphore XL (40). Université Hacettepe, Ankara, Textes réunis et publiés par Zeynep Mennan, Istanbul : Isis, 393-414.

Yilmaz, S. et Uras Yilmaz, A. (2011). Structuration et fonctionnement des dispositifs syntaxiques en turc parlé. Synergies Turquie, 4, Istanbul : Gerflint, 107-119.

Zubizarreta, M. \& Nava, E. (2011). Encoding discourse-based meaning: Prosody vs syntax. Implications for second language acquisition. Lingua, 121, 652-669. 